\title{
A life-threatening condition-ruptured ectopic pregnancy with negative urine pregnancy test: A case report
}

\author{
Fadzilah Mohamad, Ahmad Shuib Yahya, Aneesa Abdul Rashid, Navin Kumar Devaraj, \\ Abdul Hadi Abdul Manap
}

Mohamad F, Yahaya AS, Abdul Rashid A, et al. A life-threatening condition—ruptured ectopic pregnancy with negative urine pregnancy test: A case report. Malays Fam Physician. 2021;16(1);121-123. https://doi.org/10.51866/cr1117

\section{Keywords:}

ectopic pregnancy, negative

urine pregnancy test, ruptured

ectopic, reproductive women

\section{Authors:}

Fadzilah Mohamad

(Corresponding author)

MB BCH BAO (Ire), MMed Family

Medicine (UPM)

Department of Family Medicine

Faculty of Medicine \& Health

Sciences, Universiti Putra Malaysia

Selangor, Malaysia

Email: ilafadzilah@upm.edu.my

\section{Ahmad Shuib Yahya}

MBBCh (Ain Shams University),

MMed Obstetrics and Gynaecology

(USM)

Department of Obstetrics and

Gynaecology, Faculty of Medicine

\& Health Sciences, Universiti Putra

Malaysia, Selangor, Malaysia

\section{Aneesa Abdul Rashid}

MB BCH BAO (Ire), MMed Family

Medicine (UKM)

Department of Family Medicine

Faculty of Medicine \& Health

Sciences, Universiti Putra Malaysia

Selangor, Malaysia

\section{Abstract}

Ectopic pregnancy is an extra-uterine pregnancy and is a potentially life-threatening condition that can lead to death from intra-peritoneal hemorrhage. This case reports a rare occurrence of ruptured tubal pregnancy in which the patient presented early with abdominal pain and a negative urine pregnancy test but subsequently presented again with evidence of intra-peritoneal hemorrhage. A negative urine pregnancy test is often used to rule out pregnancy, but it is not $100 \%$ sensitive. Complete assessment is critical in this important diagnosis in order to plan for the appropriate emergency management.

\section{Introduction}

The incidence of ectopic pregnancy (EP) is $2 \%$ with a mortality rate of 0.5 per 100,000 live births. ${ }^{1}$ The most common site is the fallopian tube $(96 \%)$. EP is among the leading causes of maternal mortality and morbidity. ${ }^{1}$ Failure to diagnose may result in intra-peritoneal hemorrhage, shock, and death. Ruptured EP is a gynecological emergency. It should be suspected in women of reproductive age presenting with an acute abdomen. A positive urine pregnancy test (UPT) with a negative ultrasound finding of intrauterine gestational sac will increase the suspicion of EP. However, pregnancy is usually excluded when the UPT is negative and thus results in a missed diagnosis of EP. This case report illustrates how EP was missed at the primary care level, resulting in a life-threatening intra-peritoneal hemorrhage.

\section{Case Report}

A 33-year-old woman, para 1+2, presented to a primary care clinic with two days history of dull localized left lower abdominal pain. It was associated with vaginal bleeding that was thought to be her usual menses. She had an irregular menstrual cycle, and her last menstrual period was five weeks ago. She was previously on injectable contraception. Her last injection was five months ago. She had histories of dilatation and curettage for her previous miscarriages. During the first visit, no UPT was done. She was treated for dysmenorrhea and discharged home.
Three days later, she presented again for worsening abdominal pain. Her pain score was $6 / 10$. The pain radiated to the right lower abdomen. She remained afebrile and there was no vaginal bleeding. Her vital signs were stable. Abdominal examination was unremarkable except for tenderness in the lower abdomen. Transabdominal ultrasound (TAS) showed no evidence of an intrauterine gestational sac. Urinalysis was normal. UPT was negative. She was once again discharged home.

She presented to the emergency department two days later with severe generalized abdominal pain. It was associated with vomiting, abdominal distension, and dyschezia. She was tachycardic (120 beats per minute) but her other vital signs were normal. Examination revealed a distended and guarded abdomen with cervical motion tenderness. UPT was positive. She was referred to the gynecology team for suspected ruptured EP. Transvaginal ultrasound (TVS) showed an empty uterus with a $5 \times 4 \mathrm{~cm}$ left adnexal complex mass and free fluid collection in the pelvic cul-de-sac. An emergency diagnostic laparoscopy was done. Intra-operationally, the left fallopian tube was disrupted, and a left salpingectomy was performed. Postoperatively, she recuperated well.

\section{Discussion}

The classic clinical triad of EP includes abdominal pain, amenorrhea, and vaginal 


\author{
Navin Kumar Devaraj \\ MD (UPM), MMed Family Medicine \\ (UM) \\ Department of Family Medicine \\ Faculty of Medicine \& Health \\ Sciences, Universiti Putra Malaysia \\ Selangor, Malaysia
}

\section{Abdul Hadi Abdul Manap}

MD (UPM), MMed Family Medicine (UPM)

Department of Family Medicine

Faculty of Medicine \& Health

Sciences, Universiti Putra Malaysia

Selangor, Malaysia bleeding. ${ }^{1}$ UPT is a mandatory test in all women of childbearing age presenting with these symptoms. It is a quick and easy qualitative test for beta human chorionic gonadotropin $(\beta-\mathrm{hCG})$. The sensitivity is $99 \%$ if the $\beta$-hCG level is greater than 25 IU/L. ${ }^{2}$ Lower levels of $\beta$-hCG may result in a false-negative reading. The incidence of EP with a negative UPT is $1.6 \% .^{3}$ In this case study, the first issue to be highlighted is the failure of the doctor to perform UPT during the patient's initial visit despite having symptoms suggestive of EP. The missed diagnosis led to mismanagement and delays in treatment. Errors of treatment and diagnosis are common medical negligence claims brought against medical professionals. ${ }^{4}$

Discontinuation of injectable contraception is known to delay the return of fertility, sometimes up to 18 months after the last injection. ${ }^{5}$ However, in this case, fertility returned right after discontinuation and this could also be the reason why the doctor missed this crucial diagnosis.

Another issue in this case was the falsenegative UPT that misled the doctor in ruling out EP. $\beta$-hCG is detectable in urine and serum within 16 days following the luteinizing hormone surge. ${ }^{6}$ In normal pregnancies, the serum $\beta$-hCG level doubles every 48 to 72 hours but in EP, it increases at a lesser rate. ${ }^{1}$ A study done among women with EP showed that the increment of $\beta$-hCG was only $75 \%$ after 48 hours compared to $124 \%$ in a viable pregnancy ${ }^{7}$ and it declined at the same rate or faster than those with complete abortion, such that the results could be interpreted as "normal" for a complete abortion. $^{7}$ A defect in the biosynthesis of $\beta-\mathrm{hCG}$ in an ectopic trophoblast may also cause a false-negative result. The trophoblastic tissue may have been inactive before it ruptured and could have resulted in premature clearance of $\beta$-hCG from the circulation. $^{3}$ A faulty strip could be another reason 8 but this unlikely in this case as no other inaccurate UPT results were reported in the clinic during that period.

The preferred diagnostic tool for EP is TVS. ${ }^{?}$ Once suspected, serial serum $\beta$-hCG should be measured. The absence of an intrauterine pregnancy with increasing $\beta$-hCG serum above the discriminatory zone suggests an EP. ${ }^{10}$ However, at the primary care level, these procedures are usually unavailable. The doctor needs to rely on history and clinical examination. TAS is widely available in primary care; however, it has little benefit in the early stages of EP as the diagnostic reliability of TAS is only $70 \%$ compared to $\geq 90 \%$ for TVS. $^{2}$ TAS may only be able to visualize the presence of free fluid and an empty uterus. ${ }^{2}$ Therefore, the ability to perform TVS should be recommended to be part of the Credentialing and Privileging program among the primary care doctors that may aid the diagnosis of EP.

Ruptured EP is a clinical diagnosis with clinical features such as sudden and persistent abdominal pain that may be accompanied by shoulder pain, peritonitis, or shock. ${ }^{1,10}$ In this case study, the patient had a compensated hemorrhagic shock as evidenced by tachycardia, normotension, and hemoperitoneum.

This report highlights the fallibility during the early presentation of EP. A comprehensive history should always be obtained, especially the menstrual, gynecological, and contraceptive history. EP must be excluded in all sexually active women with abdominal pain regardless of the presence of vaginal bleed. The patient's history of irregular menses, recent withdrawal of contraception, and two previous dilatation and curettage procedures were indicators toward a diagnosis of EP. Early detection can reduce the risk of tubal rupture and allows more conservative treatment to be employed. ${ }^{1}$

\section{Conclusion}

$\mathrm{EP}$ is a life-threatening condition that requires urgent recognition and treatment. Although UPT is a reliable tool for detecting pregnancy, a negative result does not rule out EP. It must be suspected in a sexually active woman presenting with abdominal pain, amenorrhea, and/or vaginal bleeding.

\section{Acknowledgment}

Written consent was obtained from the patient for the publication of this case report.

\section{Funding and Conflict of Interest}

This case report did not receive any funding. All authors declare no conflict of interest in this study. 


\section{How does this paper make a difference to general practice?}

- A flaw in the diagnostic workup of this case was that the exclusion of EP was solely based on the negative UPT result.

- EP should be suspected and UPT should be mandatory in all sexually active women with lower abdominal pain, amenorrhea, and/or vaginal bleeding.

- Negative UPT with high suspicion of EP should be managed as an emergency until proven otherwise. Thorough history and examination are important and would make a considerable impact on the management of EP.

- Having TVS at the primary care level may assist in the diagnosis of EP at an earlier stage.

- Timely referral of EP to tertiary centers may prevent life-threatening complications.

\section{References}

1. Vicken PS, Ellen W. Ectopic Pregnancy. Medscape. Updated Sep 28, 2017; Accessed 9 July, 2020. Available from: https://emedicine. medscape.com/article/2041923

2. Madani Y. The use of ultrasonography in the diagnosis of ectopic pregnancy: a case report and review of the literature. Medscape J Med. 2008;10(2):35. Accessed 29 Mar, 2020.

Available from: http://www.medscape.com/ viewarticle/568521

3. Paritakul P. Ruptured tubal pregnancy with a negative urine pregnancy test and serum beta human chorionic gonadotropin. Thai J Obstet Gynaecol. 2017;25(2):130-135. doi:10.14456/ tjog.2017.19

4. Powers E. Chapter 2: Why doctors get sued. In: Jha S, Ferriman E. Medicolegal Issues In Obstetric and Gynaecology. Springer International Publishing; 2018:9-13.
5. Kaunitz AM. Depot medroxyprogesterone acetate (DMPA) for contraception: Formulations, patient selection and drug administration. In: Post T, ed. UpToDate. UpToDate; 2020. Accessed 9 Jul, 2020. Available from: https://www.uptodate.com/ contents/depot-medroxyprogesterone-acetatedmpa-for-contraception-formulations-patientselection-and-drug-administration

6. Greene DN, Grenache DG; Education Committee of the Academy of Clinical Laboratory Physicians and Scientist. Pathology consultation on human chorionic gonadotropin testing for pregnancy assessment. Am J Clin Pathol. 2015;144(6):830-836. doi:10.1309/ AJCP7O7VAREDUYIJ

7. Silva C, Sammel MD, Zhou L, Gracia C, Hummel AC, Barnhart K. Human chorionic gonadotropin profile for women with ectopic pregnancy. Obstet Gynecol. 2006;107(3):605-610. doi:10.1097/01. AOG.0000198635.25135.e7
8. Brennan DF, Kwatra S, Kelly M, Dunn M. Chronic ectopic pregnancy--two cases of acute rupture despite negative beta hCG.J Emerg Med. 2000;19(3):249-254. doi:10.1016/ s0736-4679(00)00233-x

9. Elson CJ, Salim R, Potdar N, Chetty M, Ross JA, Kirk EJ on behalf of the Royal College of Obstetricians and Gynaecologists. Diagnosis and management of ectopic pregnancy. BJOG Int J Obstet Gynaecol. 2016;123(13):e15-e55. doi:10.1111/1471-0528.14189

10. Togas T. Ectopic pregnancy: Clinical manifestations and diagnosis. In: Post T, ed. UpToDate. UpToDate; 2020. Accessed Mar 27, 2020. Available from: https://www. uptodate.com/contents/ectopic-pregnancyclinical-manifestations-and-diagnosis 